\title{
Analysis of Supergranule Sizes and Velocities Using Solar Dynamics Observatory (SDO)/Helioseismic Magnetic Imager (HMI) and Solar and Heliospheric Observatory (SOHO)/Michelson Doppler Imager (MDI) Dopplergrams
}

\author{
Peter E. Williams • W. Dean Pesnell • John G. Beck • \\ Shannon Lee
}

Received: 3 November 2012 / Accepted: 14 May 2013 / Published online: 13 June 2013

(C) Springer Science+Business Media Dordrecht 2013

\begin{abstract}
Co-temporal Doppler images from Solar and Heliospheric Observatory (SOHO)/ Michelson Doppler Imager (MDI) and Solar Dynamics Observatory (SDO)/Helioseismic Magnetic Imager (HMI) have been analyzed to extract quantitative information about global properties of the spatial and temporal characteristics of solar supergranulation. Preliminary comparisons show that supergranules appear to be smaller and have stronger horizontal velocity flows within HMI data than was measured with MDI. There appears to be no difference in their evolutionary timescales. Supergranule sizes and velocities were analyzed over a ten-day time period at a 15 -minute cadence. While the averages of the time-series retain the aforementioned differences, fluctuations of these parameters first observed in MDI data were seen in both MDI and HMI time-series, exhibiting a strong cross-correlation. This verifies that these fluctuations are not instrumental, but are solar in origin. The observed discrepancies between the averaged values from the two sets of data are a consequence of instrument resolution. The lower spatial resolution of MDI results in larger observed structures with lower velocities than is seen in HMI. While these results offer a further constraint
\end{abstract}

\footnotetext{
P.E. Williams (凶)

Department of Physics, Northern Virginia Community College, Annandale, VA, USA

e-mail: pewilliams@nvcc.edu
}

P.E. Williams

Catholic University of America, Washington, DC, USA

P.E. Williams · W.D. Pesnell

Code 671, NASA Goddard Space Flight Center, Greenbelt, MD, USA

W.D. Pesnell

e-mail: william.d.pesnell@nasa.gov

J.G. Beck

Stanford University, Stanford, CA, USA

e-mail: jbeck@solar.stanford.edu

S. Lee

San Francisco State University, San Francisco, CA, USA

e-mail: shannonl@sfsu.edu 
on the physical nature of supergranules, they also provide a level of calibration between the two instruments.

Keywords Sun: convection · Dopplergrams · Helioseismology $\cdot$ Sun: photosphere · SDO/HMI · SOHO/MDI · Sun: supergranulation

\section{Introduction}

Doppler images have proved a solid observational source from which various properties of supergranulation have been studied (Rieutord and Rincon, 2010). Leighton, Noyes, and Simon (1962) published the first Doppler images of the Sun and, from these, identified the velocity fields now attributed to supergranules. Doppler observations have been made by a variety of observatories ever since. Until recently, one of the most prominent of these has been the Michelson Doppler Imager (MDI: Scherrer et al., 1995) onboard the Solar and Heliospheric Observatory (SOHO). MDI has provided regular Dopplergrams covering the duration of Solar Cycle 23, from which a vast array of studies have been performed.

With the launch of the Solar Dynamics Observatory (SDO: Pesnell, Thompson, and Chamberlin, 2012), the Helioseismic Magnetic Imager (HMI: Scherrer et al., 2012) has taken over as the prime space-based supplier of solar Doppler observations. Table 1 summarizes a comparison of HMI and MDI specifications, highlighting the superior resolution and cadence of HMI. The line-formation heights are approximately equal for both instruments (Norton et al., 2006) and models suggest that the lines are formed at $100 \mathrm{~km}$ and $125 \mathrm{~km}$ for the HMI and MDI lines, respectively (Norton et al., 2011). Despite the small height difference of the lines, both instruments are viewing essentially the same atmospheric layer.

Leighton, Noyes, and Simon (1962) quantified a variety of supergranulation parameters. Superganule cell sizes are about $30 \mathrm{Mm}$ across, have outflow velocities of $500 \mathrm{~m} \mathrm{~s}^{-1}$, and an upper limit on their lifetime on the order of one to two days. Subsequent Doppler observations have both verified and constrained these findings (Duvall, 1980; Hathaway et al., 2000, 2002; DeRosa and Toomre, 2004; Meunier et al., 2007; Hirzberger et al., 2008). Additional verification has come from analyses of the chromospheric network (Hagenaar, Schrijver, and Title, 1997; McIntosh et al., 2011), which is produced by emission from energetic gas trapped along magnetic-field lines that have been advected by divergent supergranular flows to the boundaries of adjacent supergranules where the field lines are trapped.

Studies of supergranulation are favorable during periods of low solar activity, i.e. low sunspot number, where the supergranule pattern is uncontaminated by active regions (Rast, 2010). Comparisons of supergranule parameters during these periods of solar minimum have suggested that the properties remain virtually constant (Williams and Pesnell, 2011; McIntosh et al., 2011). However, there are suggestions that these parameters may change over

Table 1 Comparisons of SOHO/MDI and SDO/HMI instrumental parameters.

\begin{tabular}{lll}
\hline Parameter & SOHO/MDI & SDO/HMI \\
\hline Wavelength & Ni I $6768 \AA$ & Fe I $6173 \AA$ \\
Cadence & $60 \mathrm{sec}$ & $45 \mathrm{sec}$ \\
Image size & $1024 \times 1024$ & $4096 \times 4096$ \\
Field of view & 34 arcminutes & 34 arcminutes \\
Pixel resolution & 2 arcseconds & 0.5 arcseconds \\
\hline
\end{tabular}


the course of a solar cycle with, for example, an anti-correlation relationship between supergranule size and global magnetic activity, as has been reported by Meunier and Roudier (2007) and Meunier, Roudier, and Rieutord (2008).

Although originally presumed to be an element of convection (Simon and Leighton, 1964), this form of energy transport as an underlying mechanism for supergranulation loses credence due to the lack of a strong temperature profile across individual supergranule cells (Meunier, Tkaczuk, and Roudier, 2007; Goldbaum et al., 2009), unlike that seen within granules. Alternative methods for their production have been suggested. Wolff (1995) produced a model that uses energy loss from cavity-trapped oscillations within the convection zone to drive supergranule-sized flows, which manifest themselves at the solar surface. Gizon, Duvall, and Schou (2003) highlighted the observation that the supergranule pattern appears to rotate faster than the plasma in which it is embedded, suggesting a wavelike nature to supergranulation. Data simulations have shown, however, that a substantial contribution to the super-rotation derives from projecting the Doppler velocity pattern onto the line-of-sight (Hathaway, Williams, and Cuntz, 2006). However, Meunier and Roudier (2007) have shown that the super-rotation still exists, using an analysis that is unbiased by projection-effects. Meanwhile, Rast (2003) proposed a model for supergranular flow that was driven by the cumulative downflow of granules, while Rieutord et al. (2000) proposed that large-scale instabilities of the granular flow are responsible for the mechanisms driving supergranulation.

In this article, comparisons between supergranule parameters derived from HMI and MDI Doppler images are presented. Other comparisons between HMI and MDI have been made (Howe et al., 2011b; Doerr and Roth, 2011); one in particular (Howe et al., 2011a) found a satisfactory agreement in the Doppler patterns between the HMI, MDI, and GONG images.

\section{Data Preparation}

Full-disk Dopplergrams produced by HMI and MDI between 1-10 June 2010 were obtained from the Joint Science Operations Center (JSOC) data system (jsoc.stanford.edu) at Stanford University (see Table 1 for the respective observational parameters). The HMI Dopplergrams were obtained from the hmi.V_45s dataset, while those for MDI were obtained from the mdi.fd_V_lev18 dataset. Both include a wide variety of velocity components: p-mode oscillations, axi-symmetric flows (Hathaway et al., 2010), convective blueshift (Dravins, 1982), and resolved convection flows. To isolate the latter, the Dopplergrams undergo a series of subtraction processes to remove the unwanted velocity components.

To remove the five-minute $p$-mode oscillations, sets of original Dopplergrams are temporally averaged covering a time range of 31 minutes (Hathaway, 1988). To account for differential rotation, Dopplergrams are de-rotated so that their rotational phase matches that of the central image. The set is then weighted by a Gaussian function that peaks at the central image, and averaged to produce a single Dopplergram with a vastly reduced $p$-mode signal. This process is performed over the whole dataset and the resulting images are sampled to produce a data product containing a set of averaged, de-rotated Dopplergrams with a 15-minute cadence.

Each 15-minute Dopplergram is processed to remove the velocity components due to differential rotation, meridional flow, and convective blueshift (Hathaway, 1987, 1992; Williams and Pesnell, 2011). For a single image, each component is modeled with a spherical harmonic function, which is then subtracted from the data. Other signals due to the gravitational redshift and the observer motions relative to the Sun are also removed. 


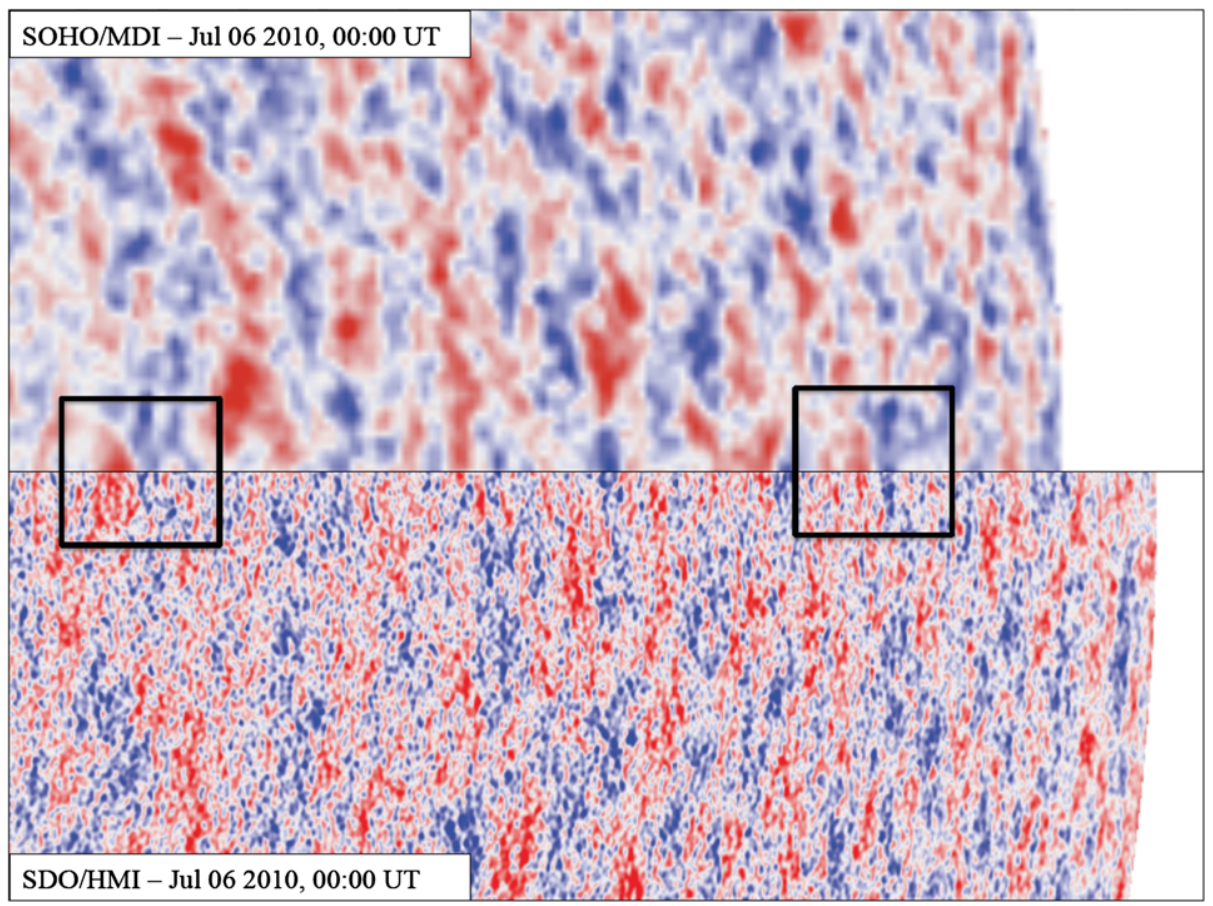

Figure 1 Cross-equatorial comparison of MDI (top) and HMI (bottom) Doppler patterns extracted from co-temporal, heliocentric images observed at 00:00 UT on 6 July 2010 . Both extractions extend from $40^{\circ} \mathrm{W}$ of the center meridian to the western edge of the respective images. The MDI extraction extends from the Equator to $10^{\circ} \mathrm{N}$, while for HMI it extends from the Equator to $10^{\circ} \mathrm{S}$. The black squares highlight regions showing the continuity across the Equator of the Doppler pattern near the western limb. The difference of coverage toward the solar limb between HMI and MDI is due to a smaller masking-radius used during the processing of the MDI images.

Finally, optical artifacts are removed that contribute to anomalous, background-velocity signals due to uncorrected observer effects and instrumental distortions, for example. Illustrative examples of these for HMI, MDI, and GONG are provided by Howe et al. (2011b). There is the strong possibility that signals due to very large-scale convective flows (diameters above $290 \mathrm{Mm}$ ) are also subtracted, but as they are not the focus of the present analysis, their removal is not considered to be important. The artifact signal is removed by remapping the Dopplergrams from image to heliographic coordinates (Thompson, 2002) and projecting onto the spherical harmonics from which the spectral amplitudes $\left[A_{\ell}^{m}\right]$ below $\ell=15$ are used to construct a Doppler map of the instrumental artifact signal, which is subtracted from the original input image. The result is remapped back to the original image coordinates. The final Doppler images in both coordinate systems consist only of velocity signals due to the convective flows.

Figure 1 shows details of MDI and HMI Doppler images after the image-reduction process. Both clearly show the horizontally diverging supergranulation flow as blue (approaching) and red (receding) patterns varying from center to limb due to the line-of-sight projection of the velocity signal. The HMI image clearly shows a more detailed pattern than that of MDI because of its superior resolution. While the patches of flow are fairly smooth in the MDI image, the HMI image exhibits consistent small-scale speckling caused by granule cells that are resolved by the instrument. The difference in the spatial extent of the image 
is due to the processing of the filtergrams that constructs the Dopplergrams. The HMI data clearly extend farther out to the limb than those of MDI because the masking-radius during the production of the MDI images is more reduced than for those produced for HMI. The continuity of the supergranule pattern observed via a cross-Equator comparison of HMI and MDI images is highlighted by the black boxes in Figure 1. The large-scale Doppler pattern perfectly matches across the Equator. This example highlights that both instruments are consistently coherent in their observations of the Sun.

\section{Data Analyses}

Contemporaneous data covering 1 - 10 June 2010 were selected for this study. This period was selected because there are very few active regions that could produce spurious velocity values within the Dopplergrams. Both data sets contain images at a 15-minute cadence produced by the temporal-averaging process described in Section 2. These images subsequently underwent the velocity-component subtraction (Section 2), resulting in sets of heliocentric and heliographic Doppler images of non-axisymmetric photospheric flows. The comparative data analyses performed on MDI and HMI data sets, including estimations of supergranule diameters, correlation lifetimes, and horizontal velocities that are described below, are similar to those performed on MDI data by Williams and Pesnell (2011).

\subsection{Supergranule-Size Analysis}

To study the size distribution of supergranule cells within a Doppler image, the globally averaged supergranule diameter is estimated from a power spectrum (Williams and Pesnell, 2011). As mentioned previously, while removing instrumental artifacts, the heliographic maps are projected onto the spherical harmonics to produce a set of spectral amplitudes $\left[A_{\ell}^{m}\right]$ in spherical harmonic degree $[\ell]$ and order $[m]$ (Hathaway, 1992). From these amplitudes, a power spectrum $[P(\ell)]$ is constructed by summing over $m$ (Hathaway et al., 2000):

$$
P(\ell)=\sum_{m=-\ell}^{\ell}\left|A_{\ell}^{m}\right|^{2} .
$$

Figure 2 (top) compares the HMI (blue) and MDI (red) power spectra averaged over the ten days of derived spectra. The plot at the top-left shows how the HMI distribution of power extends to wavenumbers beyond that of MDI because of the greater spatial resolution of the former. The plot at the top-right zooms into the MDI wavenumber range, comparing the distribution of power across the dominant supergranule feature. These features are fitted for both distributions using a function based on a Lorentzian that has been multiplied by the independent variable and subsequently squared (Williams and Pesnell, 2011). The peak of the fit is used to determine the spatially and temporally averaged supergranule diameter (Figure 2, bottom). The spectral features peaking at $\ell_{\text {peak }}^{\mathrm{MDI}}=119$ and $\ell_{\text {peak }}^{\mathrm{HMI}}=132$ relate to supergranule diameters of $36.8 \mathrm{Mm}$ and $33.2 \mathrm{Mm}$, respectively. The difference between these two average values is explained in Section 5.

\subsection{Correlation Decay Rates and 1/e Lifetimes}

Supergranules are observed to have lifetimes of typically between one and two days. Using correlation methods, Doppler patterns of supergranulation can be tracked as they move 

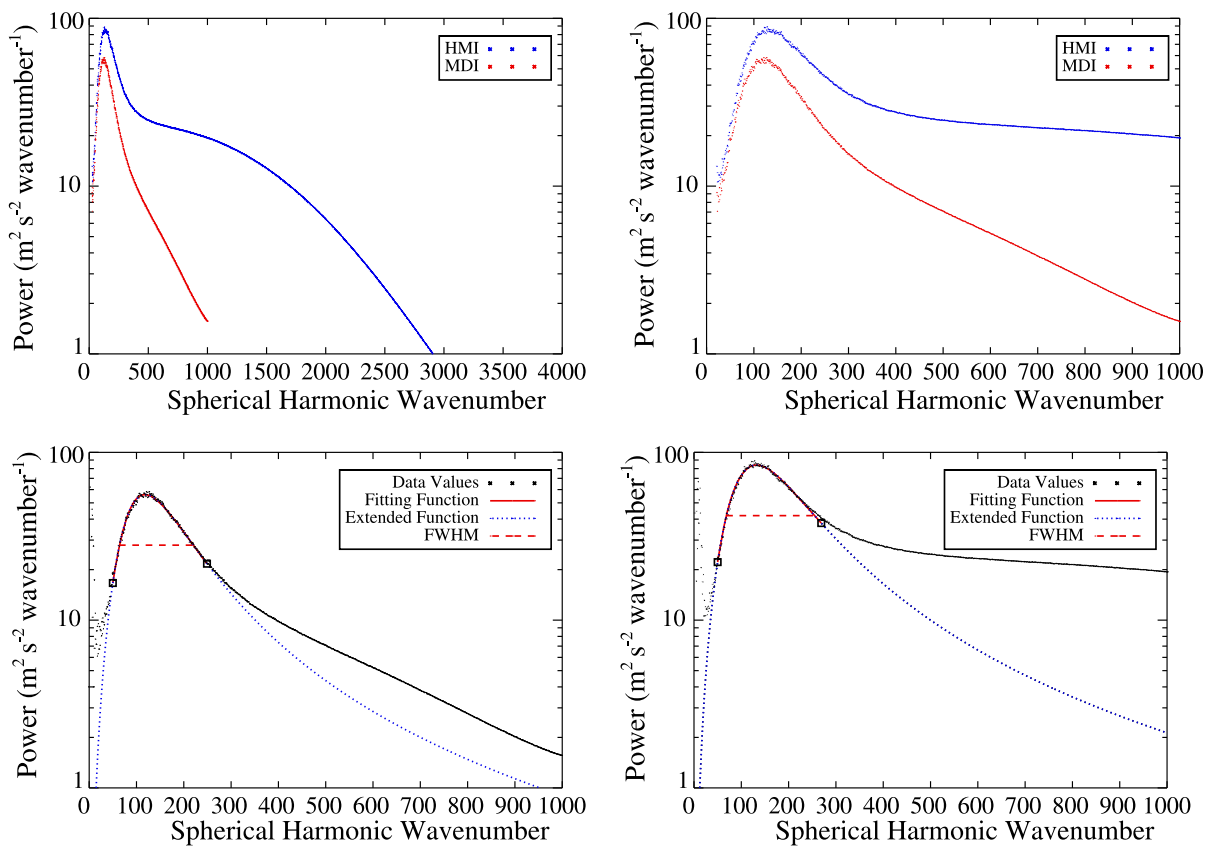

Figure 2 (Top) Comparisons of the distribution of photospheric cells sizes, resulting from spherical harmonic spectral analysis, averaged over ten days of 15 -minute cadence data covering the MDI $(\ell \leq 1000$, left $)$ and HMI ( $\ell \leq 4000$, right) wavenumber range. (Bottom) The supergranule features of the averaged spectra are fitted with a modified Lorentzian. The peak values, relating to supergranule diameter, were calculated to be $\ell_{\text {peak }}^{\mathrm{MDI}}=119$ for MDI (left) and $\ell_{\text {peak }}^{\mathrm{HMI}}=132$ for HMI (right).

across the image by solar rotation. Meanwhile, the decay of the pattern can be determined by correlating the pattern at a set time with patterns at subsequent times. The decay of the related correlation coefficient acts as a proxy for the lifetime of supergranulation.

By comparing the correlation decay rates of supergranule patterns from MDI between 1996 and 2008, Williams and Pesnell (2011) found that the 1/e lifetimes of the Doppler pattern were equal ( $\approx 18$ hours). The present study performs a similar, but more detailed, analysis.

A series of Doppler images 12 hours in length was selected from the dataset. From the first image, a strip of data, called the base strip, was extracted between $-61.6^{\circ}$ and $-8.8^{\circ}$ longitude and $0^{\circ}$ and $+8.8^{\circ}$ latitude. These correspond to $1200 \times 200$ pixel strips for HMI data and to $300 \times 50$ pixel strips for MDI data.

A secondary equal-dimension strip was extracted from the same position and correlated with the base strip to give the expected unity correlation coefficient. The secondary strips were extrapolated at incremental pixel shifts over a range of 200 pixel shifts for HMI and 50 pixel shifts for MDI. Each was correlated with the base strip to produce an array of correlation coefficients for a series of pixel shifts at zero time-lag. This process was then repeated for similar secondary strips extracted over the remaining 47 images within the 12hour dataset. The result is an array of correlation coefficients as a function of the number of pixels shifted and the time lag between the two strips. Such an array (Figure 3, top) displays a ridge of positive correlation beginning with perfect correlation at the bottom left and 

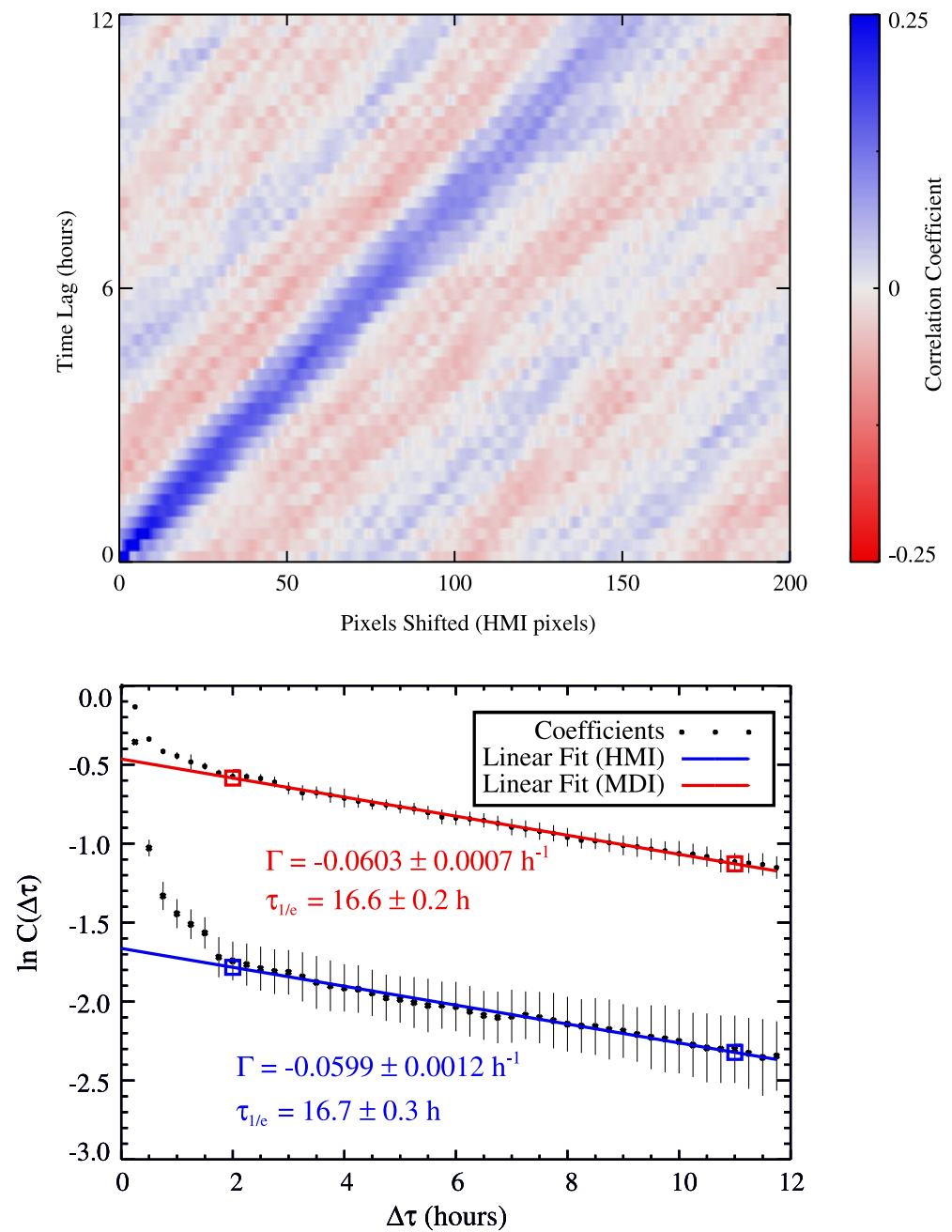

Figure 3 (Top) Correlation array for 1 June 2010 over a period of 12 hours from HMI data. The values correspond to coefficients derived from correlating two strips of Doppler data. The secondary strip originates from the full 12-hour array of images, each being shifted across the image, producing a correlation coefficient. Bluer and redder values signify more positive and more negative correlation, respectively, with a ceiling value of \pm 0.25 outside of which the colors remain at their maximum contrast. The diagonal ridge of positive correlation projecting from the bottom-left relates to the supergranule pattern contained in the first, base, strip as it advects due to solar rotation at a rate of 13.2 degrees per day. (Bottom) Plots showing the decay of the correlation coefficient in time for MDI (fitted in red) and HMI (fitted in blue). The large reductions of correlation at low lag-times is due to the evolution of the granulation pattern.

decaying with time (increasing time-lag) as the pattern is advected by rotation (increasing pixel-shift).

It is notable that as the secondary strip is selected from increasing pixel shifts from the base strip while at a constant time-lag and also when strips are extracted from subsequent images at the same position and correlated with the base strip, the correlation oscillates with a low amplitude about zero, highlighting the change in phase of the Doppler pattern 
between the base and secondary strips. Also notable is the spread and subsequent break-up of the positive correlation ridge relating to the decay of the tracked Doppler pattern.

The original Doppler pattern was tracked across the image using two methods that performed an analysis of the correlation array: One was based on correlation tracking and the other used a Radon transform. Both methods act as a cross-check that should produce the same results.

The first method extracts data around the positive correlation ridge at each time-lag by tracking the pixel shift of the ridge corresponding to the 3.4 pixel shift per image of the Doppler pattern across the Sun. These data are then fitted with a sinc-function and the amplitude of the fit calculated to be the peak correlation coefficient at that time-lag. This is performed over all time-lags for each set of 12-hour images comprising the full ten-day dataset, producing a set of 20 correlation decay plots. The data are averaged over all plots, producing a mean time-dependent correlation-decay plot.

The second method uses a Radon transform (Deans, 1983) to extract the ridge data. The array is transformed into Radon space and a mask produced at the intercept values relating to those just outside the ridge. The mask then covers the correlation array and the non-zero data at each time-lag are extracted and fitted with a sinc-function. Data averaging, as described for the previous method, is then performed.

As expected, both methods produced the same results, the Radon version is displayed in Figure 3 (bottom), which compares the correlation decays for HMI and MDI that cover the same time-period. Despite the differences between the initial drop at time-lags below two hours, the decay rates at time-lags similar to the lifetime of the supergranules are equal within their margins of error. It can be concluded that HMI and MDI exhibit identical supergranule decay characteristics with 1/e correlation-decay times of $16.7 \pm 0.3$ hours and $16.6 \pm 0.2$ hours, respectively.

It is notable, however, that while the error in the fit is similar, the error bars for each point differ. The greater error bars for HMI are due to the granulation pattern embedded into the supergranule pattern, as seen in Figure 1. This results in a broader spread of correlation values over the 20 sets (two sets per day) of decay data than for MDI.

Granulation is also the cause for the differences between the initial drop of the correlation coefficients. Within the first two hours of each analysis, the granulation pattern has significantly changed because the lifetime of individual granules is about 30 minutes. This is reflected better in the HMI data, which resolve the full range of granulation, than for MDI, which would only include the largest granules. The study of the granulation pattern decay within HMI Doppler images has been postponed to future research.

\subsection{Horizontal Velocities}

The Doppler images clearly illustrate the qualitative characteristics of supergranule flow velocities. The weak signal at the center of an image relates to a weaker radial flow than the stronger diverging horizontal flows illustrated by the strong signals toward the limb.

Using 1996 MDI Doppler images, Hathaway et al. (2002) determined these radial and horizontal velocities to be $29 \pm 2 \mathrm{~m} \mathrm{~s}^{-1}$ and $258 \pm 1 \mathrm{~m} \mathrm{~s}^{-1}$, respectively. Using a spatial filter to isolate Doppler velocities relating to supergranules with sizes around the spectral peak (cf. Section 3.1), they derived relative velocities of $13.4 \pm 1.0 \mathrm{~m} \mathrm{~s}^{-1}$ and $153.1 \pm 0.5 \mathrm{~m} \mathrm{~s}^{-1}$.

Williams and Pesnell (2011) performed a similar analysis to compare horizontal velocities between supergranulation observed in 1996 and 2008 MDI Doppler images. A lognormal filter was used to isolate the supergranule signal, and the horizontal velocities in the 1996 and 2008 data were determined to be $139 \pm 1 \mathrm{~m} \mathrm{~s}^{-1}$ and $141 \pm 1 \mathrm{~m} \mathrm{~s}^{-1}$, respectively. 
Figure 4 Comparison of the RMS horizontal velocities derived from analyses of HMI (blue) and MDI (red) Doppler images. Because the images are sensitive to limb effects, the linear fits were performed out to an angular distance of $45^{\circ}$ from disk center.

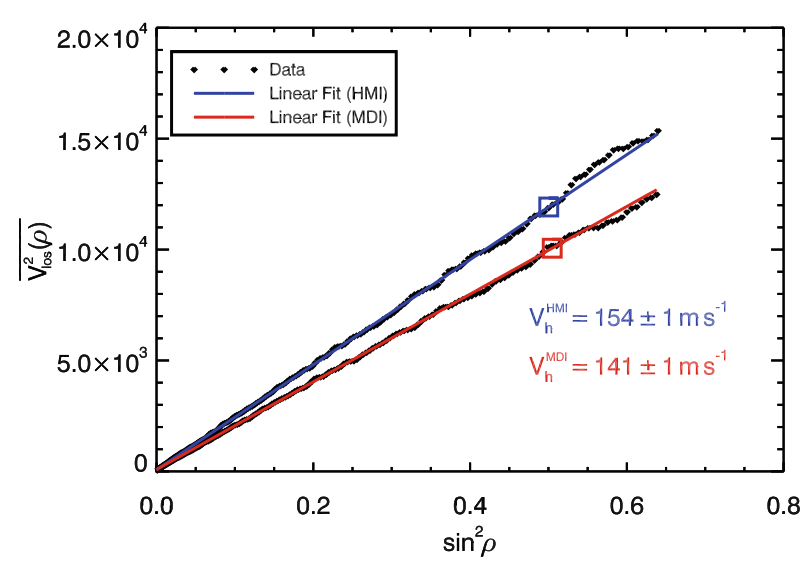

This same analysis was used to compare horizontal velocities between $2010 \mathrm{HMI}$ and MDI data.

The spatial spectrum relating to each image was filtered to isolate the supergranule component using a log-normal function

$$
F(\ell)=\frac{1}{\sigma \ell \sqrt{2 \pi}} \exp \left[\frac{-\ln ^{2}(\ell / \mu)}{2 \sigma^{2}}\right],
$$

where $\ell$ denotes the wavenumber, and $\mu$ and $\sigma$ are the mean and standard deviation of the function, respectively. From this, a new filtered image was produced. This image was then divided into 200 equal-width annuli centered on the disk center. Each annulus has an angular radius $[\rho]$ defined to be halfway between its inner and outer edge. The averagesquared line-of-sight velocity $\left[\overline{V_{\text {los }}^{2}(\rho)}\right]$ was then calculated within each annulus, and this dataset was averaged over all images within the two ten-day datasets.

A plot of $\overline{V_{\text {los }}^{2}(\rho)}$ against $\sin ^{2} \rho$ for all annuli can be fitted with a straight line whose slope is related to the horizontal velocity. Figure 4 shows data plots and their linear fits derived from the HMI and MDI Doppler images. The results show that horizontal velocities are found to be higher in the HMI data, $V_{\mathrm{h}}^{\mathrm{HMI}}=154 \pm 1 \mathrm{~m} \mathrm{~s}^{-1}$, than in the MDI data, $V_{\mathrm{h}}^{\mathrm{MDI}}=141 \pm 1 \mathrm{~m} \mathrm{~s}^{-1}$. The higher velocities measured by HMI relate to the higher spectral amplitudes in Figure 2, which we discuss in Section 5. The MDI velocity is similar to the values derived from 1996 and 2008 MDI data (Williams and Pesnell, 2011).

\section{Time-Series Comparisons}

Williams and Pesnell (2013) performed the supergranule size and velocity analysis described in Section 3 on individual 1996 and 2008 Doppler images, from which values of $\ell_{\text {peak }}$ and $V_{\mathrm{h}}$ were derived. After plotting time-series of these quantities, fluctuations on the order of three to five days were discovered. The origin of these fluctuations (solar or instrumental) was studied by performing similar analyses on the HMI and MDI datasets.

Values of $\ell_{\text {peak }}$ and $V_{\mathrm{h}}$ were derived from the full-field HMI and MDI ten-day datasets, producing four separate time-series. The supergranule sizes and velocities were then compared between the two instruments by plotting their respective co-temporal values. Figure 5 

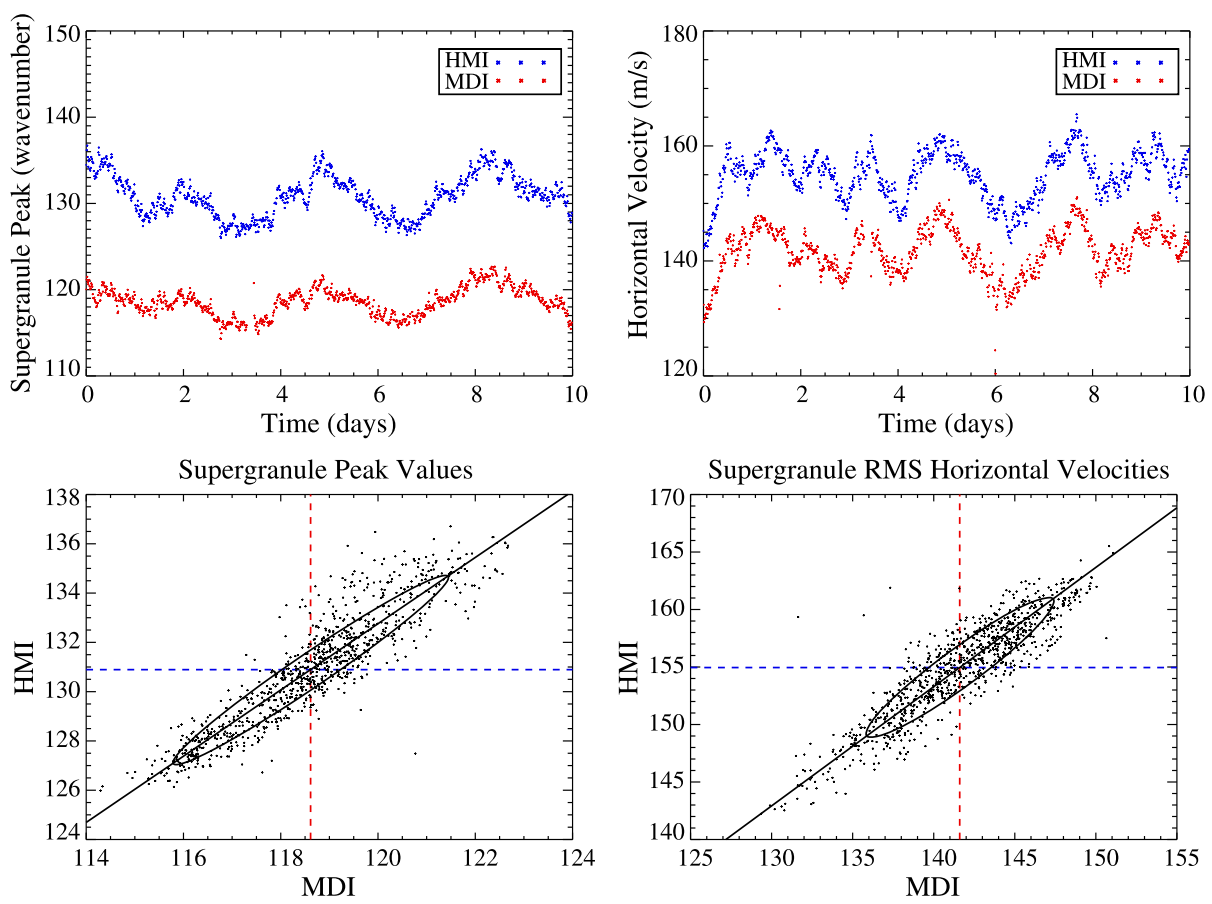

Figure 5 Comparison of HMI (blue) and MDI (red) time-series for the peak of the supergranule spectral feature (top-left) and RMS horizontal velocity (top-right). The values were derived from each Doppler image over the ten-day datasets using the methods described in the main text. The comparisons were extended to a pair of scatter plots relating to the respective parameters (bottom). Included in both correlation plots is a linear fit to the scatter along with the $1 \sigma$ error ellipse (solid black line) and the mean value for the HMI (dashed blue line) and MDI (dashed red line) data. The Pearson coefficients were calculated to be $R_{\mathrm{HMI}, \mathrm{MDI}}^{\ell_{\text {peak }}}=0.91$ and $R_{\mathrm{HMI}, \mathrm{MDI}}^{V_{\mathrm{h}}}=0.89$.

(top-left and top-right) shows the two sets of comparisons illustrating their respective qualitative correlations. Not only do large-scale $(\approx$ one - two days) variations match, but so do the embedded small-scale fluctuations. The differences in the mean values of the two timeseries are discussed in Sections 3.1 and 3.3.

To perform a quantitative comparison, the Pearson correlation coefficient was calculated for each comparative pair. A general formula to calculate the Pearson coefficient $\left[R_{x, y}\right]$ between two arbitrary time-series $x$ and $y$ consisting of $N_{x}$ and $N_{y}$ values $x_{i}$ and $y_{i}$ is given by

$$
R_{x, y}=\frac{1}{n-1} \sum_{i=1}^{n}\left(\frac{x_{i}-\bar{x}}{\sigma_{x}}\right)\left(\frac{y_{i}-\bar{y}}{\sigma_{y}}\right),
$$

where $\bar{x}, \bar{y}, \sigma_{x}$, and $\sigma_{y}$ are the means and standard deviations of the $x$ and $y$ time series, respectively. The correlation was carried out with points that were present within both timeseries. If no point existed at a given time in either series, that time-step was removed from the calculation. For the $\ell_{\text {peak }}$ values, the correlation between the two datasets is $R_{\mathrm{HMI}, \mathrm{MDI}}^{\ell_{\text {peak }}}=0.91$, while for $V_{\mathrm{h}}$, it is $R_{\mathrm{HMI}, \mathrm{MDI}}^{V_{\mathrm{h}}}=0.89$. Figure 5 (bottom-left and bottom-right) illustrates the correlations of the two pairs of datasets with a scatter-plot. The narrow linear distribution of 
the points relates to the high correlation of the pairs. This high correlation suggests that the fluctuations observed in these and other time-series are solar in nature and not instrumental artifacts. Correlations between $\ell_{\text {peak }}$ and $V_{\mathrm{h}}$ time-series have been performed for 1996 and 2008 MDI data (Williams and Pesnell, 2013). Both years exhibited anti-correlation between the two parameters. This suggested a positive correlation between supergranule size and horizontal velocity, agreeing with previous work (Meunier et al., 2007).

\section{Discussion, Conclusion, and Future Work}

Doppler data taken from contemporaneous HMI and MDI observations of the Sun were analyzed to reveal the global properties of supergranulation. Within the images, the high resolution of HMI allows granulation to be observed, which is distributed within the supergranule Doppler pattern. Comparing the supergranule patterns side-by-side shows the very good continuity between the two. Comparisons of supergranule decay rates, sizes, and velocities were performed, as well as correlations of the latter two quantities between the two instruments (Figure 5, cf. Section 4). The results, with their relevant one- $\sigma$ uncertainties, are compiled in Table 2.

While the spherical harmonic decomposition of HMI images exhibits power at granule scales, this research focused on comparing supergranule features between HMI and MDI power spectra, notably the wavenumber at which the feature peaks. We found that the feature peaks at higher wavenumbers for HMI than for MDI, suggesting that HMI observes smaller supergranule cells. This consequently provides a tighter constraint on the depth that supergranule cells penetrate into the convection zone, and also suggests a higher density of the magnetic-field lines that are congregated around the supergranule boundaries.

By correlating a strip of Doppler data with a second strip shifted spatially and temporally, the decay of the supergranule pattern could be measured. While the HMI decay exhibited a deeper initial drop than seen by MDI, the supergranule pattern decay was observed to have the same evolution within both the HMI and MDI data due to the decay of the now-resolved granule pattern.

By measuring the distribution of line-of-sight velocities over a Doppler image, it was possible to estimate the RMS horizontal velocities. It was found that these velocities are higher for HMI than for MDI. This suggests a higher rate of mass transport from the supergranule centers to their boundaries and subsequently a more rapid advection of magnetic-field lines toward the supergranule edges, where they congregate to produce the chromospheric network. It would be of interest for future work to analyze the advection rates of individual magnetic-field lines toward supergranule boundaries using Ca II $\mathrm{K}$ images to compare the

Table 2 Compiled results comparing supergranule parameters derived from HMI and MDI Doppler images.

\begin{tabular}{lll}
\hline Parameter & SDO/HMI & SOHO/MDI \\
\hline 1/e Lifetime & $18.8 \pm 1.2 \mathrm{hr}$ & $17.6 \pm 0.5 \mathrm{hr}$ \\
Peak $\ell$ & $132 \pm 1$ & $119 \pm 1$ \\
Diameter & $33.2 \pm 0.3 \mathrm{Mm}$ & $36.8 \pm 0.3 \mathrm{Mm}$ \\
Corr. Coeff & 0.91 & $141 \pm 2 \mathrm{~m} \mathrm{~s}^{-1}$ \\
Horiz. Velocity & $154 \pm 2 \mathrm{~m} \mathrm{~s}^{-1}$ & \\
Corr. Coeff & 0.89 & \\
\hline
\end{tabular}


horizontal velocities within supergranule cells from these images and from Doppler measurements.

Performing the size and velocity analysis on individual Doppler images across both sets of data, it was possible to qualitatively and quantitatively compare the respective HMI and MDI time-series. Their distributions appear to be very similar for large- as well as smallscale fluctuations. Their similarities are apparent when performing a cross-correlation for the two quantities between the HMI and MDI data because of the resulting high values of the Pearson correlation coefficients. Meanwhile, the discrepancies between the mean values of the correlated datasets are retained.

These discrepancies were investigated to understand their cause. Using the hypothesis that the cause was related to the difference in the optical resolution of the two instruments, the HMI images were smoothed to mimic the optical resolution of the MDI image. Two-dimensional Lorentzian functions of different widths were used to smooth a base de-rotated Dopplergram. We reduced the Dopplergram contributions to those of the convective flows and performed a spherical harmonic transform, the result of which yielded a one-dimensional power spectrum as in Figure 2.

Figure 6 (left) shows two examples of a derived power spectrum after their parent image (1 June 2010; 00:00 UT) was smoothed by a 2D Lorentzian filter with a FWHM of 5 and 13 pixels along with the spectrum of the unsmoothed image. Two observations are made: i) power at high wavenumbers decreases as the width of the smoothing function increases, thus decreasing the resolution of the smoothed image, and ii) the peak of the supergranule feature shifts to lower wavenumbers. To illustrate the latter point in more detail, the smoothing process was performed for a series of filters with different widths and the wavenumber at which the supergranule feature peaks from each resulting spectrum plotted against the FWHM of the filter (Figure 6, right).

Both results are due to the smearing of the Doppler image that spreads the cellular pattern, which produces a higher estimate of the size of the supergranules that is observed in the discrepancy between the values of $\ell_{\text {peak }}$ for HMI and MDI. Using Figure 6 (right), a
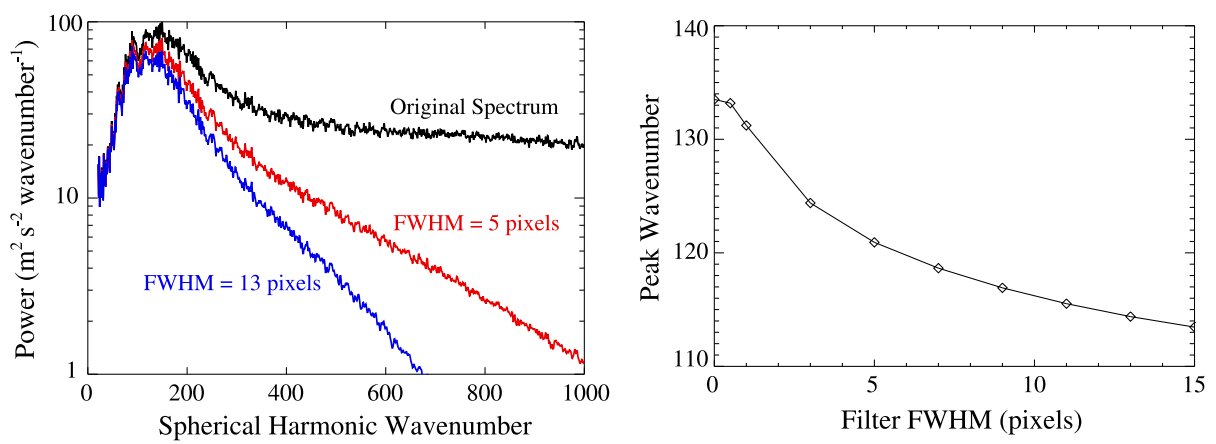

Figure 6 (Left) Spectral distribution of photospheric cell sizes from a single HMI Doppler image before (black) and after smoothing the image with a 2D Lorentzian function with a FWHM of 5 (red) and 13 (blue) pixels, which replicates the image resolution reduction. Clearly, the high-wavenumber spectral power drops for lower resolutions, related to smaller features falling below the resolution threshold. However, also seen is a related shift in the peak wavenumber of the supergranule feature. This is illustrated for a larger selection of filter widths (right) where the evolution of feature peak to higher wavenumbers is apparent for broader filter widths (i.e. lower resolutions). This explains the discrepancy in the values of $\ell_{\text {peak }}$ and $V_{\mathrm{h}}$ between HMI and MDI and suggests that the supergranule sizes previously determined by MDI could have been too large while the horizontal velocities could have been too low due to the smoothing-out of the signal over the image. 
six-pixel FWHM filter would be required to reduce the resolution of the HMI to the respective MDI image. This single-image result is close to the seven-pixel FWHM filter used by Howe et al. (2011a) to compare HMI, MDI, and GONG Doppler images. The discrepancy in the velocities follows because the Doppler-velocity signal has been smeared out by the lower resolution of MDI, producing lower velocities. This is mirrored by the lower amplitudes at all wavenumbers in the power spectra of Figure 6 (left). These observations lead to two conclusions with regard to supergranule observations made with HMI and MDI: HMI observes i) higher supergranule velocities and ii) smaller supergranule sizes than MDI. This suggests a previous underestimation and overestimation by MDI of supergranule velocities and sizes, respectively, using similar analysis techniques. In addition to the consequences mentioned above, these results also provide further constraints that may be applied to numerical simulations that model supergranular flows and the magnetic fields that are advected within. Direct comparisons of our quantitative results with those derived from other observations and analysis methods are difficult without a unified investigation that would bring these observational and analytical differences on an equal footing. Additionally, other solarbased factors that have been found to cause variations in supergranule parameters may have to be taken into consideration, such as global solar activity (Meunier, Roudier, and Rieutord, 2008; McIntosh et al., 2011), the vicinity of local active regions (DeRosa and Toomre, 2004), and latitudinal dependence (Raju and Singh, 2002).

It is noted from the HMI spectra (Figure 2) that although granules are now resolved by the instrument, they do not contribute much power. This is because i) most granules, with lifetimes of $\approx$ one hour, are removed by the temporal filtering early in the data-reduction phase, and ii) the area where granule flows would dominate, near the center of the Doppler image because of their strong radial upflows, are relatively small compared with the image as a whole. The majority of the image, away from disk center, contains flows diverging from the supergranulation, as seen in Figure 1, which contributes to most of the power in the spectrum. Analyses of the granule signal will be performed, with results to be published in a future article.

Fluctuations in supergranule parameters previously seen only in MDI data (Williams and Pesnell, 2013) are now verified to have a solar origin because they are also present in HMI, which is supported by the high correlation between the respective datasets. While mechanisms that could cause these fluctuations are as yet unknown, they could be related to the wave phenomena originally observed by Gizon, Duvall, and Schou (2003) or to the superposition of random processes occurring in the Sun that influence supergranule parameters. There may, of course, be another alternative mechanism yet to be understood.

Acknowledgements This research was supported by an appointment to the NASA Postdoctoral Program at NASA Goddard Space Flight Center, administered by Oak Ridge Associated Universities through a contract with NASA via the Solar Dynamics Observatory. SDO is part of NASA's Living With a Star (LWS) program. HMI was designed and assembled at Stanford University and Lockheed Martin Solar and Astrophysics Laboratory. SOHO is a project of international cooperation between ESA and NASA. The authors wish to thank the referee for the comments that further enhanced the contents of this article.

\section{References}

Deans, S.R.: 1983, The Radon Transform and Some of Its Applications, Wiley, New York.

DeRosa, M.L., Toomre, J.: 2004, Evolution of solar supergranulation. Astrophys. J. 616, $1242-1260$. doi:10.1086/424920.

Doerr, H.-P., Roth, M.: 2011, Localized averaging kernels for probing the deep meridional flow with data from GONG, MDI and HMI. J. Phys. CS-271(1), 012057. doi:10.1088/1742-6596/271/1/012057. 
Dravins, D.: 1982, Photospheric spectrum line asymmetries and wavelength shifts. Annu. Rev. Astron. Astrophys. 20, 61 - 89. doi:10.1146/annurev.aa.20.090182.000425.

Duvall, T.L. Jr.: 1980, The equatorial rotation rate of the supergranulation cells. Solar Phys. 66, $213-221$. doi:10.1007/BF00150578.

Gizon, L., Duvall, T.L., Schou, J.: 2003, Wave-like properties of solar supergranulation. Nature 421, 43 - 44. doi:10.1038/nature01287.

Goldbaum, N., Rast, M.P., Ermolli, I., Sands, J.S., Berrilli, F.: 2009, The intensity profile of the solar supergranulation. Astrophys. J. 707, 67 - 73. doi:10.1088/0004-637X/707/1/67.

Hagenaar, H.J., Schrijver, C.J., Title, A.M.: 1997, The distribution of cell sizes of the solar chromospheric network. Astrophys. J. 481, 988. doi:10.1086/304066.

Hathaway, D.H.: 1987, Spherical harmonic analysis of steady photospheric flows. Solar Phys. 108, 1-20. doi:10.1007/BF00152073.

Hathaway, D.H.: 1988, Temporal filters for isolating steady photospheric flows. Solar Phys. 117, $1-12$. doi:10.1007/BF00148567.

Hathaway, D.H.: 1992, Spherical harmonic analysis of steady photospheric flows. II. Solar Phys. 137, 15 - 32. doi:10.1007/BF00146573.

Hathaway, D.H., Williams, P.E., Cuntz, M.: 2006, Supergranule superrotation identified as a projection effect. Astrophys. J. 644, 598-602. doi:10.1086/498842.

Hathaway, D.H., Beck, J.G., Bogart, R.S., Bachmann, K.T., Khatri, G., Petitto, J.M., Han, S., Raymond, J.: 2000, The photospheric convection spectrum. Solar Phys. 193, 299-312. ADS:2000SoPh..193..299H, doi:10.1023/A:1005200809766.

Hathaway, D.H., Beck, J.G., Han, S., Raymond, J.: 2002, Radial flows in supergranules. Solar Phys. 205, 25 - 38. ADS:2002SoPh..205...25H, doi:10.1023/A:1013881213279.

Hathaway, D.H., Williams, P.E., Dela Rosa, K., Cuntz, M.: 2010, The advection of supergranules by the Sun's axisymmetric flows. Astrophys. J. 725, 1082 - 1090. doi:10.1088/0004-637X/725/1/1082.

Hirzberger, J., Gizon, L., Solanki, S.K., Duvall, T.L.: 2008, Structure and evolution of supergranulation from local helioseismology. Solar Phys. 251, 417 - 437. doi:10.1007/s11207-008-9206-8.

Howe, R., Jain, K., Hill, F., Komm, R., González Hernández, I., Bogart, R.: 2011a, Comparison of HMI Dopplergrams with GONG and MDI data. J. Phys. CS-271(1), 012060. doi:10.1088/1742-6596/ 271/1/012060.

Howe, R., Tripathy, S., González Hernández, I., Komm, R., Hill, F., Bogart, R., Haber, D.: 2011b, Ring-diagram parameter comparisons for GONG, MDI and HMI. J. Phys. CS-271(1), 012015. doi:10.1088/1742-6596/271/1/012015.

Leighton, R.B., Noyes, R.W., Simon, G.W.: 1962, Velocity fields in the solar atmosphere. I. Preliminary report. Astrophys. J. 135, 474. doi:10.1086/147285.

McIntosh, S.W., Leamon, R.J., Hock, R.A., Rast, M.P., Ulrich, R.K.: 2011, Observing evolution in the supergranular network length scale during periods of low solar activity. Astrophys. J. Lett. 730, L3. doi:10.1088/2041-8205/730/1/L3.

Meunier, N., Roudier, T.: 2007, The superrotation of solar supergranules. Astron. Astrophys. 466, 691 -696. doi:10.1051/0004-6361:20066790.

Meunier, N., Roudier, T., Rieutord, M.: 2008, Supergranules over the solar cycle. Astron. Astrophys. 488, 1109 - 1115. doi:10.1051/0004-6361:20078835.

Meunier, N., Tkaczuk, R., Roudier, T.: 2007, Intensity variations inside supergranules. Astron. Astrophys. 463, 745 - 753. doi:10.1051/0004-6361:20066314.

Meunier, N., Tkaczuk, R., Roudier, T., Rieutord, M.: 2007, Velocities and divergences as a function of supergranule size. Astron. Astrophys. 461, 1141 - 1147. doi:10.1051/0004-6361:20065625.

Norton, A.A., Graham, J.P., Ulrich, R.K., Schou, J., Tomczyk, S., Liu, Y., Lites, B.W., López Ariste, A., Bush, R.I., Socas-Navarro, H., Scherrer, P.H.: 2006, Spectral line selection for HMI: a comparison of Fe I $6173 \AA$ and Ni I $6768 \AA$ A. Solar Phys. 239, 69-91. doi:10.1007/s11207-006-0279-y.

Norton, A.A., Graham, J.P., Ulrich, R.K., Schou, J., Tomczyk, S., Liu, Y., Lites, B.W., López Ariste, A., Bush, R.I., Socas-Navarro, H., Scherrer, P.H.: 2011, On the formation height of the SDO/HMI Fe I $6173 \AA$ Doppler signal. Solar Phys. 271, 27 -40. doi:10.1007/s11207-011-9783-9.

Pesnell, W.D., Thompson, B.J., Chamberlin, P.C.: 2012, The Solar Dynamics Observatory (SDO). Solar Phys. 275, 3 - 15. doi:10.1007/s11207-011-9841-3.

Raju, K.P., Singh, J.: 2002, Dependence of supergranular length-scales on network magnetic fields. Solar Phys. 207, 11 - 16. ADS:2002SoPh..207...11R, doi:10.1023/A:1015585010078.

Rast, M.P.: 2003, Supergranulation: new observation, possible explanation. In: Sawaya-Lacoste, H. (ed.) GONG+ 2002. Local and Global Helioseismology: The Present and Future SP-517, ESA, Noordwijk, $163-172$.

Rast, M.P.: 2010, Is there such a thing as quiet Sun? In: Cranmer, S.R., Hoeksema, J.T., Kohl, J.L. (eds.) SOHO-23: Understanding a Peculiar Solar Minimum CS-428, Astron. Soc. Pac., San Francisco, 87. 
Rieutord, M., Rincon, F.: 2010, The Sun's supergranulation. Living Rev. Solar Phys. 7(2). doi:10.12942/ Irsp-2010-2.

Rieutord, M., Roudier, T., Rincon, F., Malherbe, J.-M.: 2000, On mesogranulation, network formation and supergranulation. Astron. Astrophys. 357, 1063.

Scherrer, P.H., Bogart, R.S., Bush, R.I., Hoeksema, J.T., Kosovichev, A.G., Schou, J., Rosenberg, W., Springer, L., Tarbell, T.D., Title, A., Wolfson, C.J., Zayer, I., MDI Engineering Team: 1995, The solar oscillations investigation - Michelson Doppler imager. Solar Phys. 162, 129-188. doi:10.1007/BF00733429.

Scherrer, P.H., Schou, J., Bush, R.I., Kosovichev, A.G., Bogart, R.S., Hoeksema, J.T., Liu, Y., Duvall, T.L., Zhao, J., Title, A.M., Schrijver, C.J., Tarbell, T.D., Tomczyk, S.: 2012, The Helioseismic and Magnetic Imager (HMI) investigation for the Solar Dynamics Observatory (SDO). Solar Phys. 275, 207 -227. doi:10.1007/s11207-011-9834-2.

Simon, G.W., Leighton, R.B.: 1964, Velocity fields in the solar atmosphere. III. Large-scale motions, the chromospheric network, and magnetic fields. Astrophys. J. 140, 1120. doi:10.1086/148010.

Thompson, W.T.: 2002, Standardized coordinate systems for solar image data. Adv. Space Res. 29, 2093 2098. doi:10.1016/S0273-1177(02)00155-2.

Williams, P.E., Pesnell, W.D.: 2011, Comparisons of supergranule characteristics during the solar minima of Cycles 22/23 and 23/24. Solar Phys. 270, 125 - 136. doi:10.1007/s11207-011-9718-5.

Williams, P.E., Pesnell, W.D.: 2013, Time-series analysis of supergranule characteristics at solar minimum. Solar Phys., accepted.

Wolff, C.: 1995, Oscillation-convection coupling: cause of supergranulation. Astrophys. J. 443, 423-433. doi:10.1086/175535. 